\title{
VALIDEZ FACTORIAL, CONSISTENCIA INTERNA Y \\ REPRODUCIBILIDAD DE LA ESCALA DE SEGURIDAD ALIMENTARIA EN HOGARES DE BUCARAMANGA, COLOMBIA
}

\author{
FACTORIAL VALIDITY, INTERNAL CONSISTENCY AND \\ REPRODUCIBILITY OF THE SCALE OF FOOD SECURITY \\ IN HOUSEHOLD OF BUCARAMANGA, COLOMBIA
}

Oscar Fernando Herrán F. (1, 2), Doris Cristina Quintero L. (2), Gloria Esperanza Prada G. $(1,2)$

(1) Escuela de Nutrición y Dietética. Universidad Industrial de Santander, Colombia

(2) Observatorio Epidemiológico de Enfermedades Cardiovasculares. Centro de Investigaciones Epidemiológicas.

Universidad Industrial de Santander, Bucaramanga, Colombia.

\begin{abstract}
The objective of this study was to assess in households of Bucaramanga, the factorial validity, internal consistency and reproducibility of the scale of perception of food security (EPSA) applied in the Survey of the Nutritional Situation in Colombia (ENSIN-2005). During the first semester of 2007, a questionnaire with socioeconomic variables was applied in 108 household and, with interval of one month, EPSA was applied twice. Factor analysis and goodness of fit of the final model and the theoretical one were evaluated. We established the reproducibility of the EPSA and its internal consistency. We ratified a dimension with two components; ilack of money to buy foodsî and iexperience of hunger for lack of foods.î This model is similar to the one applied in the ENSIN-2005, explains $76 \%$ of the variance and its adjustment was barely acceptable: RMSEA 0.27, NFI 0.63$, GFI 0.58. The reproducibility was almost perfect ( $r=0.98, C I ; 0.98-0.99)$ and had internal validity, alpha of Cronbach 0.92.
\end{abstract}

Key words: Food security; reproducibility of results; validity of the tests; hunger; Colombia.

Este trabajo fue recibido el 23 de Diciembre de 2008 y aceptado para ser publicado el 20 de Marzo de 2009.

\section{INTRODUCCIÓN}

El aumento sostenido de la pobreza en Latinoamérica y el Caribe durante los últimos 20 años, como de sus nuevas expresiones, aceleradas en este siglo debido al aumento de los precios del petróleo, de los alimentos básicos, y de la especulación con el capital (1-3), ha llevado nuevamente a los gobiernos y agencias internacionales a centrar sus esfuerzos para garantizar a nivel de país, hogar e individuo, un mínimo de alimentos que permita llevar en cada nivel un estilo de vida productivo y saludable (4). Esta preocupación ha facilitado recrear el término "seguridad alimentaría", el cual en una versión amplia incluye además del derecho a la alimentación, el del acceso al agua potable (4-6).

La seguridad alimentaria (SA) tradicionalmente se ha medido en el ámbito de un país con "hojas de balance" de alimentos y en poblaciones con estudios del consumo dietario en hogares e individuos (7). De manera indirecta la SA se estima a través del costo de la canasta básica alimentaria o el estado de nutrición (7). Más recientemente la SA a nivel de hogar se ha estimado con escalas simplificadas (8-23). En los últimos 25 años éstas han sido utilizadas para estimar el riesgo, la magnitud y la severidad de la inseguridad alimentaria (IA) en el ámbito del hogar. También para focalizar programas de ayuda gubernamental, evaluar su impacto (24), e incluso para simular modelos en los cuales se sustenta la política pública (25).

Las escalas más utilizada en Latinoamérica y el Caribe, son derivadas de la propuesta por Wehler en 1992 para obtener un índice de hambre (8). En Colombia, una versión adaptada y validada factorialmente en una 
de las 42 regiones geopolíticas que la componen (26), fue aplicada en la Encuesta Nacional de la Situación Nutricional 2005 (ENSIN-2005) (27).

La investigación social y epidemiológica, incluida la realizada en nutrición, se basa en buena medida en instrumentos de medición denominados encuestas, escalas ó instrumentos, éstas pueden ser extensas y complejas ó simplificadas, y pretenden la mayoría de las veces, reemplazar a otros métodos en teoría más confiables, para hacer la investigación viable en términos de tiempo y costo. Tal vez el principal problema al diseñar o reemplazar métodos de medición por otros menos complejos, es que la capacidad discriminatoria de éstos puede verse comprometida, llevando a mala clasificación y sesgos que diluyen o distorsionan las relaciones causales (28). El resultado de una encuesta depende de muchos factores, entre otros de la prevalencia del evento bajo estudio, del orden de aplicación del instrumento dentro de una batería de pruebas, del orden de las preguntas, de la relación simbólica que establecen los sujetos con el evento, y otras valoraciones sociales y culturales complejas e incluso desconocidas (29). Tradicionalmente, un instrumento antes de su aplicación debe ser sometido a adaptaciones lingüísticas, a estudios de reproducibilidad, y de validación de diferentes tipos (30). Un instrumento considerado útil en un contexto, no lo es necesariamente en otro (29).

El objetivo de este estudio fue establecer en hogares de Bucaramanga, la validez factorial, la consistencia interna y la reproducibilidad de la escala de percepción de seguridad alimentaria (EPSA), utilizada en la ENSIN-2005.

\section{MATERIAL Y MÉTODOS}

Este estudio se clasifica como de evaluación de tecnología diagnóstica, se desarrolló con base en los datos recolectados en una encuesta poblacional que tuvo como objeto establecer el desempeño de la EPSA en los hogares urbanos de Bucaramanga, Colombia. Bucaramanga es la quinta ciudad el país y la más desarrollada del nor oriente colombiano.

Selección de hogares y tamaño de la muestra. Los hogares fueron seleccionados por muestreo aleatorio estratificado en múltiples etapas. Las manzanas de la ciudad se clasificaron en seis estratos socioeconómicos de acuerdo con la metodología de la oficina de planeación municipal, tres manzanas fueron seleccionadas aleatoriamente en cada estrato y sus mapas actualizados, las viviendas en ellas fueron numeradas en orden consecutivo y en las elegidas al azar, se indagó por la persona encargada de la compra de los alimentos, en un sentido más amplio, por el que responde por la alimentación del hogar. Ciento ocho sujetos adultos, representando a 108 hogares constituidos con dos años o más años de anterioridad, respondieron a dos instrumentos mediante entrevista directa, uno sobre aspectos biológicos y socioeconómicos de él y el hogar, y en dos ocasiones a la EPSA, con intervalo de un mes para borrar del recuerdo las respuestas. La muestra fue sobreestimada en $10 \%$ para compensar las pérdidas durante el seguimiento y obtener coeficientes de correlación (r) de mínimo 0,40 entre dos aplicaciones de la EPSA (Alfa $=0,05$, poder $=0,90$ ). El estudio fue aprobado por el Comité de Ética en Investigación de la Facultad de Salud de la Universidad Industrial de Santander. Todos los participantes dieron su consentimiento informado por escrito.

Escala de Percepción de Seguridad Alimentaria (EPSA). La escala evaluada fue la misma aplicada en la Encuesta Nacional de Seguridad Alimentaria (ENSIN-2 005), pero a diferencia de ésta que hace referencia al último mes, la aplicada en este estudio hizo referencia a los últimos seis meses. Consta de catorce preguntas, doce que permiten establecer el nivel de seguridad alimentaria y la severidad de la IA en el hogar, la severidad se establece con base en la sumatoria de las doce primeras preguntas y es relativa a la presencia de menores de 18 años en el hogar. Los dos últimos ítems permiten establecer el riesgo de IA en los hogares clasificados con seguridad alimentaria.

Los puntos de corte para establecer la IA y su severidad son los mismos utilizados en la ENSIN-2 005 (Apéndice 1).

Análisis estadístico. Para describir las categorías de las variables biológicas (VB) y sociodemográficas (VSE) se calcularon medidas descriptivas y sus intervalos de confianza al 95\% (IC). Para establecer si existían diferencias estadísticamente significativas entre las VB y VSE por la clasificación de seguridad o inseguridad alimentaria, se calcularon pruebas t de student, pruebas de ANOVA y de $\mathrm{Ji}^{2}$.

Para establecer la estructura factorial de la escala tanto en la primera como en la segunda aplicación y verificarla contra la teórica existente, se condujo análisis de factores. En los modelos fijados, el número de factores se estableció como consecuencia de valores "Eigen" mayores a uno. Los ítems fueron agrupados dentro de ellos si su carga "Loadings" era mínimo de 0,55 , garantizando así su significancia estadística en un tamaño muestral como el estudiado (alfa $=0,01)(31)$. Después de realizar la agrupación de variables dentro de factores se crearon nuevas bases de datos para verificar la pertinencia de la reducción de variables a través del calculo del estadístico Kaiser-Meyer-Olkin (KMO) $(31,32)$. El subgrupo de variables que obtuvo el mejor balance, entre la varianza 
explicada, el número de factores y el estadístico KMO, se consideró un modelo. Estos modelos fueron valorados a través de ecuaciones estructurales (MEE) (31). Con el fin de comparar el modelo teórico (Modelo C) con los modelos derivados A y B, fueron diseñados MEE y calculados diferentes índices de bondad de ajuste; razón de verosimilitud $\left(\mathrm{X}^{2}\right)$, error de aproximación cuadrático medio (RMSEA), el índice Tucker-Lewis (NNFI), de ajuste normal (NFI), de bondad de ajuste (GFI) y el índice de ajuste de parsimonia (PNFI) (19). Todas las soluciones factoriales, fueron realizadas con la matriz de covarianza y la estimación de la máxima verosimilitud (Likelihood) $(31,32)$. Con el coeficiente $r$ de Pearson, se estableció la correlación entre factores y el puntaje total alcanzado entre aplicaciones.

Para determinar la consistencia interna de la EPSA, además del cálculo del coeficiente Alfa de Cronbach (AC), se estimaron los coeficientes de correlación interítem, los coeficientes de correlación para cada ítem versus los restantes (33) y medidas descriptivas para cada pregunta de la EPSA y el puntaje acumulado. La reproducibilidad (test-retest) entre las dos aplicaciones de la EPSA fue estimada a través del cálculo de coeficientes de correlación de Spearman (rs) y Pearson (r) y sus respectivos IC, entre el puntaje alcanzado para cada ítem y el total acumulado. Además, se calcularon coeficientes de acuerdo Kappa de Cohen's, que son equivalentes a coeficientes de correlación intraclase (34). Para este coeficiente se estimó su máximo valor posible (Kappa Máxima) (35). Los coeficientes de Kappa fueron ajustados por el sesgo entre observadores (KSO) y la prevalencia de inseguridad alimentaria (KPA) (36). En términos de Egglin et al. (1996), los índices de acuerdo Kappa, fueron ajustados por las características del contexto (37).

Todos los registros fueron digitados por duplicado y limpiados en el programa VALIDATE de EpiInfo 6,04d (38). Los MEE fueron realizados con BDMP 7,0 (39). El procesamiento de datos, junto con el análisis fue realizado con STATA 10,1 SE (40).

\section{RESULTADOS}

Durante el primer semestre de 2007, ciento ocho sujetos residentes en el área urbana de Bucaramanga, Colombia, respondieron en dos ocasiones mediante entrevista directa la EPSA con intervalo entre aplicaciones de 30,6 días (IC; 29,2 a 32,0). Un total de 18 hogares por cada estrato socioeconómico original fue encuestado. Debido a que los valores estimados no se modificaron al corregir por el efecto del diseño de la muestra (41), los reportados son similares a los obtenidos de un muestreo aleatorio simple.
Características de los informantes. La edad media de los informantes fue de 45,8 años (IC; 43,7 a $47,9)$. Noventa y nueve fueron mujeres $(91,7 \%)$, de ellas $79(79,8 \%)$ eran madres, seis hombres eran padres. El $51,8 \%$ eran casados, el $8,3 \%$ separados y el $3,7 \%$ viudos. El 7,4\% no tiene ningún tipo de seguridad social y el $74,1 \%$ aportan como trabajadores formales o informales al sistema de seguridad social en salud. Durante los últimos seis meses 27 (25\%) sujetos consiguieron empleo y 24 sujetos $(22,2 \%)$ lo perdieron, de los que lo perdieron, el $66,7 \%$ logró emplearse nuevamente antes de seis meses. El 12\% reconoció haber incurrido en prácticas en contra de sus principios y/o integridad personal para conseguir dinero o alimentos, ocho de estos 13 sujetos $(61,5 \%)$ pertenecen al primer nivel socioeconómico y 4 (31\%) al nivel 2. El 64,8\% de los informantes manifestó que durante los últimos seis meses el dinero disponible en el hogar disminuyó.

Características de los hogares. En promedio cada hogar esta conformado por 4,5 miembros (IC; 4,1 a 4,8 ), de los cuales 0,4 son menores de siete años (IC; 0,3 a 0,6). En el 13,9\% de ellos conviven menores de 18 años. La escolaridad media del jefe del hogar fue de 10 años (IC; 9 a 11), el 2,8\% son analfabetas, el 15,7\% alcanzó la primaria y el 23,1\% el grado de bachiller. En el 42,6\% de los hogares el índice de dependencia supera el nivel crítico, más de tres personas dependiendo económicamente de una. En el 8,3\% de los hogares existía hacinamiento, ocho de los nueve casos pertenecían al primer nivel socioeconómico. El número medio de miembros que come fuera de la casa durante tres o más días a la semana era de 0,6 (IC; 0,3 a 0,8). En el 3,7\% de los hogares durante los últimos seis meses nació un niño, y en el 5,6\% murió un integrante. En el 13\% de ellos, se sumo un nuevo integrante y el $11,1 \%$ un miembro se fue del hogar o dejó de pertenecer al grupo familiar. La tenencia de la vivienda en el $52,8 \%$ era propia sin deuda, en el 13,9\% propia con deuda, en el 30,6\% casa o apartamento en arriendo y el $2,7 \%$ restante es habitación arrendada.

Inseguridad alimentaria. En las dos aplicaciones de la EPSA se evidenció IA en 22 hogares $(20,4 \%)$, en 19 de ellos $(86,4 \%)$ está se clasificó como leve o sin hambre, en dos $(9,1 \%)$ como moderada con hambre y solo en uno $(4,5 \%)$ como severa con hambre. En el $95,4 \%$ de los hogares con IA se manifestó disminución del dinero en el hogar en los últimos seis meses. En todos los niveles socioeconómicos se evidenció IA, $54,6 \%$ en el nivel uno, $27,3 \%$ en el nivel dos y $18,1 \%$ en el nivel tres, $\mathrm{p}=0,051$. La condición de IA no fue diferencial dado el nivel de dependencia económica, $\mathrm{p}=0,079$, de hacinamiento, $\mathrm{p}=0,313$ o del número de 
menores de siete años, $\mathrm{p}=0,234$, pero si por el número de integrantes en la familia, $\mathrm{p}=0,024$; diferencia media de $-1,0$ (IC; $-1,90$ a -0.13 ) y la escolaridad del jefe del hogar, $\mathrm{p}=0,002$; diferencia media de 3,8 años (IC; 1,3 a 6,0). En la primera aplicación de la EPSA en el 40,7\% de los hogares sin inseguridad alimentaria se evidenció

\section{TABLA 1}

Validez factorial; criterios para la determinación del número de factores y la reducción de variables.

Primera Aplicación

\begin{tabular}{|c|c|c|c|c|c|c|c|c|}
\hline \multirow[b]{2}{*}{$\begin{array}{l}\text { No. * } \\
\text { Factores }\end{array}$} & \multicolumn{4}{|c|}{ Primera Aplicación } & \multicolumn{4}{|c|}{ Segunda Aplicación } \\
\hline & $\begin{array}{l}\text { Varianza (\%) } \\
\text { Explicada }\end{array}$ & $\begin{array}{l}\text { No. } \\
\text { Variables }\end{array}$ & $\begin{array}{l}\mathrm{KMO} \dagger \\
\text { Variables }\end{array}$ & $\begin{array}{l}\text { KMO } \dagger \\
\text { Factores }\end{array}$ & $\begin{array}{l}\text { Varianza (\%) } \\
\text { Explicada }\end{array}$ & $\begin{array}{l}\text { No } \\
\text { Variables }\end{array}$ & $\begin{array}{l}\mathrm{KMO} \dagger \\
\text { Variables }\end{array}$ & $\begin{array}{l}\mathrm{KMO} \dagger \\
\text { Factores }\end{array}$ \\
\hline 1 & 63 & 12 & 0,58 & & 0,73 & 12 & 0,80 & \\
\hline 2 & 76 & 12 & 0,58 & 0,50 & 0,85 & 12 & 0,80 & 0,50 \\
\hline 3 & 88 & 12 & 0,60 & 0,70 & & & & \\
\hline
\end{tabular}

\section{TABLA 2}

Validez factorial; agrupación de ítems en tres modelos derivados de la EPSA.

\begin{tabular}{|c|c|c|c|c|c|c|c|}
\hline \multirow[b]{2}{*}{ Ítems } & \multicolumn{2}{|c|}{ Modelo A } & \multicolumn{3}{|c|}{ Modelo B } & \multicolumn{2}{|c|}{ Modelo C } \\
\hline & F1 & F2 & F1 & F2 & F3 & F1 & F2 \\
\hline Pregunta 1 & $\mathrm{x}$ & & $\mathrm{x}$ & & & $\mathrm{x}$ & \\
\hline Pregunta 2 & $\mathrm{x}$ & & $\mathrm{x}$ & & & $\mathrm{x}$ & \\
\hline Pregunta 3 & $\mathrm{X}$ & & $\mathrm{X}$ & & & $\mathrm{X}$ & \\
\hline Pregunta 4 & $\mathrm{X}$ & & $\mathrm{X}$ & & & $\mathrm{X}$ & \\
\hline Pregunta 5 & $\mathrm{x}$ & & $\mathrm{x}$ & & & $\mathrm{x}$ & \\
\hline Pregunta 6 & & $\mathrm{X}$ & & & $\mathrm{X}$ & & $\mathrm{X}$ \\
\hline Pregunta 7 & & $\mathrm{x}$ & & & $\mathrm{X}$ & & $\mathrm{x}$ \\
\hline Pregunta 8 & $\mathrm{X}$ & & $\mathrm{X}$ & & & $\mathrm{x}$ & \\
\hline Pregunta 9 & $\mathrm{x}$ & & & $\mathrm{X}$ & & $\mathrm{x}$ & \\
\hline Pregunta 10 & $\mathrm{X}$ & & $\mathrm{X}$ & & & $\mathrm{x}$ & \\
\hline Pregunta 11 & & $\mathrm{X}$ & & $\mathrm{x}$ & & & $\mathrm{X}$ \\
\hline Pregunta 12 & & $\mathrm{X}$ & & $\mathrm{X}$ & & & $\mathrm{X}$ \\
\hline Pregunta 13 & $\mathrm{x}$ & & $\mathrm{x}$ & & & & \\
\hline Pregunta 14 & $\mathrm{x}$ & & $\mathrm{x}$ & & & & \\
\hline $\begin{array}{l}\text { F; Factor. } \\
\text { Modelo A; Con bas } \\
\text { Modelo B; Con bas } \\
\text { Modelo C; Con bas } \\
\text { y "experiencia de h }\end{array}$ & $\begin{array}{l}\text { nda a } \\
\text { era ap } \\
\text { nensic } \\
\text { alta d }\end{array}$ & $\begin{array}{l}\text { estab } \\
\text { tos". }\end{array}$ & le d & on & tos" & & \\
\hline
\end{tabular}


modificación en la compra de alimentos relacionada con la disminución de disponibilidad de dinero, que puede considerarse riesgo de inseguridad alimentaria, el riesgo en la segunda aplicación fue de 45,4\%, p=0,000.

Validez factorial. El análisis de factores evidenció que en las dos aplicaciones, un modelo con dos factores es el que mejor ajusta. La varianza explicada por estos modelos en la primera aplicación fue de $76 \%$ y en la segunda del 85\%, (tabla 1). El estadístico KMO muestra que la pertinencia en la agrupación de las variables es pobre, mientras que para los factores es apenas aceptable, sugiriendo altas correlaciones entre ítems, y que algunos de ellos podrían eliminarse o fusionarse. La tabla 2 presenta tres modelos -agrupación de ítems en factores-, los dos derivados del análisis factorial con las preguntas utilizadas en la ENSIN-2 005 y otro que sustenta teóricamente la EPSA. La tabla 2 además, presenta la forma como se agruparon las preguntas dentro de los factores.

Al evaluarse los modelos derivados del análisis de factores y el teórico a través de MEE, se encontró que el ajuste para todos es apenas aceptable. El valor de p para el $\mathrm{X}^{2}$ de la razón de verosimilitud en los tres modelos fue menor de 0,05. El valor ideal del RMSEA es por debajo de 0,08. Para los demás índices, un valor de cero refleja un pobre ajuste y un valor de 1 , un ajuste perfecto $(31,32)$. La tabla 3 resume estos hallazgos y evidencia el desempeño de las soluciones factoriales presentadas. El desempeño del modelo teórico con dos factores que sustenta la EPSA; "falta de dinero para comprar alimentos" y "experiencia de hambre por falta de alimentos", no es diferente en términos de ajuste, al de tres y dos factores derivados matemáticamente. El modelo derivado del análisis factorial con base en los resultados de la segunda aplicación y sin añadir las preguntas trece y catorce utilizadas en la ENSIN-2 005 -Modelo A-, es igual al teórico -Modelo C-ver tablas 2 y 3. La correlación entre factores para el Modelo A fue de 0,59 (IC; 0,46 a 0,70), para el Modelo B entre F1 y F2; 0,50 (IC; 0,35, 0,63), F1 y F3 0,92 (IC; 0,88 a 0,94) y entre F2 y F3 0,55 (IC; 0,40 a 0,67), para el Modelo C entre F1 y F2 de 0,60 (IC; 0,47 a 0,71).

Estudio de reproducibilidad. El coeficiente de correlación (rs) entre las preguntas 1, 2, 3, 11 y 12 fue perfecto, de 1,0. Para todas las demás preguntas puede considerarse como muy bueno, mínimo de 0,72 (IC; 0,61 a 0,80 ) para la pregunta nueve y máximo de 0,96 (IC; 0,94 a 0,97) para la pregunta cinco. La correlación para las preguntas 13 y 14 fue igualmente alta, pregunta trece 0,89 (IC; 0,84 a 0,92 ) y pregunta catorce 0,91 (IC; $0,87$ a 0,94$)$. El coeficiente de correlación para el puntaje total alcanzado entre aplicaciones de la escala (r) fue de 0,98 (IC; 0,98 a 0,99). El coeficiente de acuerdo Kappa crudo entre aplicaciones para todas las preguntas puede considerarse como bueno y muy bueno, mínimo 0,71 para la pregunta nueve y de 1,0 para las preguntas 1,2 ,

\section{TABLA 3}

\section{Validez factorial: valores alcanzados en las medidas de bondad de ajuste para tres modelos desarrollados.}

\begin{tabular}{|c|c|c|c|}
\hline $\begin{array}{l}\text { No. de factores (No. de variables) } \\
\text { Estadístico }\end{array}$ & $\begin{array}{l}\text { Modelo A } \\
2 \text { (14) }\end{array}$ & $\begin{array}{l}\text { Modelo B } \\
3 \text { (14) }\end{array}$ & $\begin{array}{l}\text { Modelo C } \\
2 \text { (12) }\end{array}$ \\
\hline Razón de verosimilitud $\left(\mathrm{X}^{2}\right) *$ & 546 & 429 & 465 \\
\hline Error de aprox. cuadrático medio (RMSEA) & 0,24 & 0,24 & 0,27 \\
\hline Índice Tucker-Lewis (NNFI) & 0,61 & 0,58 & 0,55 \\
\hline Índice de ajuste Normal (NFI) & 0,66 & 0,65 & 0,63 \\
\hline Índice de bondad de ajuste (GFI) & 0,58 & 0,62 & 0,58 \\
\hline Índice de ajuste de parsimonia (PNFI) & 0,42 & 0,52 & 0,39 \\
\hline \multicolumn{4}{|c|}{$\begin{array}{l}\text { * Para todos valor de } \mathrm{p}<0,05 \text {. } \\
\text { Modelo A; Con base en la segunda aplicación. } \\
\text { Modelo B; Con base en la primera aplicación. } \\
\text { Modelo C; Con base en dos dimensiones preestablecidas, "falta de dinero para comprar alimentos", "experiencia de } \\
\text { hambre por falta de alimentos". }\end{array}$} \\
\hline
\end{tabular}


3, 11 y 12. La Kappa ajustada para todas las preguntas estuvo por encima de 0,90 , tabla 4.

Consistencia interna. La tabla 5 presenta la media y desviación estándar alcanzada en cada pregunta de la EPSA, también los valores alcanzados en el Alfa de Cronbach, éste muestra que la escala es unidimensional -con dos componentes- y que la consistencia se mantiene entre aplicaciones. El AC en la primera aplicación fue de 0,92 y en la segunda de 0,94 . Como se esperaba se evidenciaron altas correlaciones entre el ítem y el resto -Ítems-Rest- y entre ítems -Inter-Ítem-. La consistencia interna según AC para el primer factor en los Modelos A y $\mathrm{C}$ es de 0,92 , y para el segundo factor de 0,86 . Como se esperaba para los adultos la experiencia de hambre se presento sólo en los hogares con IA moderada y severa. Sin embargo, dos de las experiencias de hambre en menores $(50 \%)$, fue reportada en dos hogares clasificados como con IA leve.

\section{DISCUSIÓN}

En Colombia, Bucaramanga es la ciudad donde más se ha estudiado la dieta con métodos considera- dos referentes de oro $(42,43)$, lo que ha permitido, el desarrollo de instrumentos de medición simplificados para ser utilizados en la investigación epidemiológica y aproximaciones clásicas a la prevalencia de IA (4446). En estas circunstancias estudiar el desempeño de la EPSA cobra aún más relevancia. Este es el cuarto estudio de validación de la EPSA que se realiza en diferentes contextos colombianos, el primero fue realizado en hogares rurales y urbanos del departamento de Antioquia (26), el segundo en una población afrocolombiana del departamento del Cauca (47) y el tercero como parte de la evaluación de un programa de suplementación (24). Ninguno de ellos estableció la reproducibilidad de la EPSA.

Validez factorial de la EPSA. El análisis de factores evidencio que dos factores explican el $76 \%$ y $85 \%$ de la varianza en la primera y segunda aplicación respectivamente, la pertinencia de la agrupación de las preguntas dentro de ellos según el estadístico KMO es apenas aceptable, lo que sugiere que aún es posible eliminar preguntas de la escala, aspecto ya sugerido anteriormente (24) y corroborado por el valor obtenido

\section{TABLA 4}

Estudio de reproducibilidad; acuerdo alcanzado entre dos aplicaciones de la EPSA.

\begin{tabular}{lllllll}
\hline Ítem & \% Si & Kappa* & KapMax $\dagger$ & Razón $\neq$ & KSO I & KPA** \\
\hline Pregunta 1 & 20,4 & 1,000 & 1,000 & 100,0 & 1,000 & 1,000 \\
Pregunta 2 & 7,4 & 1,000 & 1,000 & 100,0 & 1,000 & 1,000 \\
Pregunta 3 & 11,1 & 1,000 & 1,000 & 100,0 & 1,000 & 1,000 \\
Pregunta 4 & 3,7 & 0,791 & 1,000 & 79,1 & 0,790 & 0,963 \\
Pregunta 5 & 11,1 & 0,955 & 1,000 & 95,5 & 0,955 & 0,981 \\
Pregunta 6 & 2,8 & 0,852 & 1,000 & 85,2 & 0,852 & 0,981 \\
Pregunta 7 & 3,7 & 0,791 & 1,000 & 79,1 & 0,790 & 0,963 \\
Pregunta 8 & 10,2 & 0,947 & 1,000 & 94,7 & 0,947 & 0,981 \\
Pregunta 9 & 4,6 & 0,713 & 0,788 & 90,4 & 0,713 & 0,944 \\
Pregunta 10 & 6,5 & 0,928 & 1,000 & 100,0 & 0,928 & 0,981 \\
Pregunta 11 & 3,7 & 1,000 & 1,000 & 100,0 & 1,000 & 1,000 \\
Pregunta 12 & 3,7 & 1,000 & 1,000 & 100,0 & 1,000 & 1,000 \\
Pregunta 13 & 39,8 & 0,886 & 0,959 & 92,4 & 0,886 & 0,889 \\
Pregunta 14 & 29,6 & 0,908 & 1,000 & 90,8 & 0,908 & 0,926 \\
Riesgo IA †† & 40,7 & 0,906 & 1,000 & 90,6 & 0,906 & 0,907 \\
\hline * Kappa de Cohen. † Máximo valor posible para Kappa de Cohen. & & & \\
† Razón (\%) entre Kappa de Cohen y Kappa máxima. & & &
\end{tabular}


en el índice de parsimonia al evaluar mediante MEE los modelos establecidos. Es importante resaltar que utilizando diferentes métodos de análisis -Factores, Componentes Principales y Rasch- se ha llegado a conclusiones similares sobre la estructura factorial, la EPSA es unidimensional con dos componentes; "falta de dinero para comprar alimentos" y "experiencia de hambre por falta de alimentos".

Reproducibilidad de la EPSA. En pocos estudios es posible observar coeficientes de reproducibilidad como los alcanzados entre las dos aplicaciones de la EPSA, estos pueden considerarse perfectos para las doce preguntas de la escala y los indicadores complementarios -riesgo de inseguridad alimentaria e índice de hambre-, éstos aspectos en la investigación epide- miológica se traducirán en estabilidad en las medidas de efecto absolutas y relativas, situación deseable al establecer modelos causales con la EPSA. Sin embargo, la estabilidad entre aplicaciones podría enmascarar las fluctuaciones y adaptaciones al interior del hogar, que en términos de seguridad alimentaria se dan necesariamente en condiciones extremas y agudas, como las del desplazamiento forzado o las de desastres naturales, eventos cada vez más comunes en la población colombiana. Este aspecto planteado anteriormente $(16,20,23)$ y que aún no ha sido investigado suficientemente, puede afectar la sensibilidad de la escala para captar cambios agudos en el hogar y por consiguiente comprometer su utilidad para focalizar ayuda humanitaria, establecer la política pública e incluso evaluar el impacto a corto plazo de

\section{TABLA 5}

Consistencia interna entre ítems de la EPSA.

\section{Primera Aplicación*}

Ítem†

Correlación Correlación AlfaJ

Media DS Ítem-Rest + Inter-Ítem $\S$ Cronbach Media DS

Pregunta

Pregunta 2

Pregunta 3

Pregunta 4

Pregunta 5

Pregunta 6

Pregunta 7

Pregunta 8

Pregunta 9

Pregunta 10

Pregunta $11 \quad 0,04$

Pregunta $12 \quad 0,04$

Pregunta $13 \quad 0,40$

Pregunta $14 \mid \quad 0,30$

Todas**

$8,41 \quad 6,91$

0,722

0,796

0,832

0,574

0,672

0,576

0,578

0,809

0,583

0,729

0,627

0,628

0,494

0,597

0,470

\section{Segunda Aplicación*}

Correlación Correlación AlfaJ

Ítem-Rest + Inter-Ítem Cronbach

$\begin{array}{llllll}0,918 & 0,20 & 0,40 & 0,765 & 0,720 & 0,934 \\ 0,915 & 0,07 & 0,26 & 0,855 & 0,826 & 0,931 \\ 0,914 & 0,11 & 0,32 & 0,859 & 0,830 & 0,931 \\ 0,923 & 0,06 & 0,23 & 0,671 & 0,613 & 0,937 \\ 0,920 & 0,12 & 0,33 & 0,822 & 0,787 & 0,932 \\ 0,923 & 0,04 & 0,19 & 0,734 & 0,684 & 0,935 \\ 0,923 & 0,06 & 0,23 & 0,803 & 0,765 & 0,933 \\ 0,915 & 0,09 & 0,29 & 0,831 & 0,798 & 0,932 \\ 0,922 & 0,06 & 0,29 & 0,713 & 0,661 & 0,936 \\ 0,918 & 0,07 & 0,26 & 0,852 & 0,822 & 0,931 \\ 0,921 & 0,04 & 0,19 & 0,638 & 0,576 & 0,938 \\ 0,921 & 0,04 & 0,19 & 0,722 & 0,671 & 0,936 \\ 0,925 & 0,43 & 0,49 & 0,525 & 0,451 & 0,942 \\ 0,922 & 0,26 & 0,44 & 0,673 & 0,616 & 0,937 \\ 0,925 & 8,45 & 6,64 & & 0,525 & 0,939\end{array}$

* Para todos las preguntas n=108. $\dagger$ Para todas Mínimo=0 (No), Máximo=1 (Si).

$¥$ Correlación entre el ítem y el resto de ítems.

$\S$ Promedio de la correlación inter-ítem. I te student entre aplicaciones ( $\mathrm{p}<0,05)$.

I Alfa de Cronbach, si se eliminará esa pregunta de la prueba.

** Con base en los primero 12 ítems -índice de inseguridad alimentaria-, valores medios de la EPSA para los hogares clasificados

como en inseguridad alimentaria;

$\mathrm{t}$ de student para medias globales alcanzadas entre aplicaciones $(n=22 ; \mathrm{p}=0,928)$.

$\mathrm{t}$ de student para Alfa de Cronbach alcanzadas entre aplicaciones $(\mathrm{p}=0,941)$. 
programas alimentarios. No debe olvidarse que la EPSA ha sido aplicada indagando por períodos de tiempo que van desde el último mes hasta los últimos doce meses (18-24, 26, 27, 47).

Consistencia interna de la EPSA. El AC alcanzado en las dos aplicaciones da cuenta de la unídimensionalidad de la escala y de la consistencia de cada pregunta al aporte del índice de seguridad alimentaria, hallazgos ya reportados en otros estudios realizados en América Latina y Colombia $(8-27,47)$. Las correlaciones entre preguntas de la escala permiten evidenciar patrones de respuesta particulares en cada contexto sociocultural, el reportado aquí no es el mismo de los otros estudios realizados en Colombia, sugiriendo mecanismos de adaptación en el hogar diferenciales entre regiones de un mismo país $(16,22,24,26)$. La correlación Item-Rest aumentó en la segunda aplicación para todas las preguntas con excepción de la número 11, sin embargo esto no afectó la clasificación final, el puntaje medio del índice de seguridad alimentaria, ni su variabilidad.

Relación con otras variables y resultados. Aún cuando no era el objetivo de este estudio establecer la validez de constructo de la EPSA con base en la relación teórica con otras variables, se evidenció que los resultados sobre IA se comportaron como se esperaba -relación inversa-, con el nivel socioeconómico, el número de integrantes del hogar y la escolaridad del jefe del mismo. En los hogares con menores se evidenció hambre en la clasificación de severidad leve, lo cual no es consistente con el constructor teórico, lo que puede llevar a mala clasificación diferencial -hogar con menores versus hogar sin menores.

Un aspecto importante ya advertido y aún no resuelto sobre la EPSA es sobre ¿cuál es su comportamiento frente a otros métodos en el ámbito del hogar o del individuo? $(16,20,23)$. La magnitud estimada de IA aquí es similar a la observada en un estudio sobre el índice de precios de los alimentos para evaluar la IA realizado en el 2000 , en hogares del primer nivel socioeconómico de la misma población, $-54,6 \%$ versus $55,1 \%$ - (46), lo anterior obliga a indagar si los resultados obtenidos por la EPSA en población general, son similares a los obtenidos con base en el costo de la canasta básica alimentaria vigilada regularmente por el Departamento Administrativo Nacional de Estadísticas (DANE), de ser así, aplicar la EPSA en hogares colombianos de población general seria un esfuerzo duplicado para llegar a conclusiones iguales que las derivadas de la información aportada por el DANE. La ENSIN-2005 (27), mostró que para la región oriental -donde se ubica Bucaramanga-, la IA fue del 47,2\%, este resultado incluye un $25 \%$ de hogares rurales y por lo tanto no es comparable con el aquí presentado. En el ámbito del individuo, utilizando métodos considerados como referentes y con base en el consumo medio de energía, la IA en los hombres de esta población es del $29,5 \%$ y en las mujeres del $33,4 \%$. Mientras no se establezca la relación entre los niveles de medición -hogar e individuo-, o si el individuo que responde a la EPSA representa realmente el comportamiento del hogar o de otros integrantes del mismo $(16,20,23)$, será difícil establecer si los resultados de la EPSA además de discriminar entre hogares, permiten establecer la magnitud real de la IA en ellos.

Utilidad del instrumento y para tomar decisiones en política pública. Desde el desarrollo del índice de hambre (8) y los posteriores trabajos realizados para adaptarlo y validarlo en America Latina que han derivado en la EPSA, se ha demostrado consistentemente sus propiedades psicométricas, ahora su reproducibilidad, y se ha avanzado substancialmente sobre algunos conceptos proximales de la IA. Sin embargo, a pesar de que la EPSA ha sido utilizada incluso en encuestas nacionales, aún no puede considerarse que el conocimiento sobre la utilidad y limitaciones de la EPSA este concluido. La EPSA es sin lugar a dudas una escala atractiva, de bajo costo y fácil de aplicar e interpretar, sin embargo sus resultados deben utilizarse conservadoramente mientras la EPSA se sigue sometiendo al escrutinio científico para contestar las preguntas aquí planteadas y otras aún por resolver.

Es claro que la EPSA permite discriminar entre hogares, pero también lo es, en que esto no traduce necesariamente que las magnitudes de IA establecidas se correspondan con las reales en los hogares y población, pues hasta ahora no se han realizado estudios de validez de la EPSA frente a referentes o estándares de oro. Los estudios de validez factorial, consistencia interna y de reproducibilidad hasta ahora realizados no reemplazan los de validez frente a un estándar de oro que involucre la ingesta energética del hogar. Escalas simplificadas, con buen desempeño factorial, consistencia interna y reproducibilidad, pueden ser no validas frente a un referente. Hasta no tener estudios complementarios la EPSA debe considerarse como una prueba en desarrollo, y por lo tanto, no es recomendable utilizar los resultados de la EPSA para tomar decisiones en temas de política pública, asignar recursos, reemplazar o incluso descartar otras metodologías alternas de medición de la seguridad alimentaría en cualquier nivel. El uso valido que se le puede dar a los resultados de EPSA -alcances y limitaciones- y el como se relacionan éstos con otros métodos que permiten estimar la IA, debe ser el centro de las futuras investigaciones. 


\section{RESUMEN}

El objetivo fue establecer en hogares de Bucaramanga, la validez factorial, la consistencia interna y la reproducibilidad de la escala de percepción de seguridad alimentaria (EPSA), aplicada en la Encuesta de la Situación Nutricional en Colombia (ENSIN-2005). Durante el primer semestre de 2 007, en 108 hogares se aplicó un formato con variables socioeconómicas, y con intervalo de un mes, dos veces la EPSA. Se realizó análisis de factores y se evaluó la bondad de ajuste del modelo final y el teórico. Se estableció la reproducibilidad de la EPSA y su consistencia interna. Se ratifico una dimensión con dos componentes; "falta de dinero para comprar alimentos" y "experiencia de hambre por falta de alimentos". Este modelo es igual al aplicado en la ENSIN-2005, explica 76\% de la varianza y su ajuste es apenas aceptable; RMSEA: 0,27, NFI: 0,63, GFI: 0,58 . La reproducibilidad es casi perfecta, $\mathrm{r}=0,98$ (IC; 0,98 a 0,99) y tiene validez interna, Alfa de Cronbach 0,92 . A pesar de las propiedades psicométricas y ahora de su reproducibilidad, aún no puede considerarse que el conocimiento sobre la utilidad y limitaciones de la EPSA este concluido.

Palabras claves: Seguridad alimentaria; reproducibilidad de resultados; validez de las pruebas; hambre; Colombia.

Dirigir la correspondencia a:

Profesor

Oscar Fernando Herrán Falla. ND. MSc.

Carrera 32 No. 29-31. Facultad de Salud.

CIE. Tercer Piso.

Universidad Industrial de Santander.

Bucaramanga.

Colombia. Sur América.

Tele fax; (57-7) 6345781 / 6323215

e-mail: herran@uis.edu.co oscar.herran@gmail.com

Declaración de conflicto de interés: Los autores declaran que no tienen conflicto de interés de ningún tipo, ni real o potencial sobre los resultados presentados.

\section{BIBLIOGRAFÍA}

1. Banco Mundial. Graeme Wheeler. Efectos del precio del petróleo en los países en desarrollo. Disponible en; http://web.worldbank.org/WBSITE/EXTERNAL/BANCOMUNDIAL/NEWSSPANISH/0,,co ntentMDK:21679096 pagePK:64257043 piPK:4 37376 theSitePK:1074568,00.html. Consultado en Octubre 5 de 2008.

2. Carestía de alimentos y petróleo deja 700.000 nue- vos pobres en Guatemala. Disponible en: http:// noticias.terra.com/articulo/html/act1316785.htm. Consultado en Octubre 5 de 2008.

3. Morales JC. Los agrocombustibles y la amenaza del hambre. En: Universidad Nacional de Colombia. Observatorio de Seguridad alimentaria y Nutricional (OBSAN). I foro académico (Memorias). Bogota, Colombia. Universidad Nacional, 2007:85-101.

4. Sen A. Desarrollo y libertad. Bogotá, Colombia. Editorial Planeta, 2001.

5. Leisinger KM, Schmitt KM, Pandya-Lorch R. Assuring food security for a growing population. In: Six billion and counting; Population and food security in the 21st century. Washington, D.C. Johns Hopkins University Press, 2002.

6. Organización de las Naciones Unidas para la Agricultura y la Alimentación. The estate of food insecurity in the World, 2006. Disponible en: http:// www.fao.org/docrep/009/a0750e/a0750e00.htm. Consultado en Octubre 5 de 2008.

7. Madrigal-Fritsch M, Salgado-Martínez H (Editores). Manual de encuestas de dieta. Perspectivas en salud pública, No. 23. Cuernavaca, México. Instituto Nacional de Salud Pública, 1996.

8. Wehler CA, Scott RI, Anderson JJ. The Community childhood identification proyect: A model of domestic hunger-demonstration project in Seattle, Washington. J Nutr (Editorial) 1992; 24: 29S35S.

9. Frongillo EA. Validation of measures of food insecurity and hunger. J Nutr 1999;129:506S-9S.

10. Carlson SJ, Andrews MS, Bickel GW. Measuring food insecurity and hunger in the United States: Development of a national benchmark measure and prevalence Estimates. J Nutr 1999; 129: 510S-6S.

11. Hamelin AN, Habicht JP, Beaudry M. Food insecurity: Consequences for the household and broader social implications. J Nutr 1999; 129:525S-8S.

12. Gary B, Nord M, Price C, Hamilton W, Cook J. Guide to measuring household food security, revised 2000. Alexandria VA: U.S. Department of Agriculture, Food and Nutrition Service, 2000.

13. Lorenzana P, Danjur D. La adaptación y validación de una escala de seguridad alimentaria en una comunidad de Caracas, Venezuela. Arch Latinoamer Nutr 2000;50:334-40.

14. Radimer KL. Measurement of household food security in the USA and other industrialized countries. Public Health Nutr 2002;5:859-64.

15. Melgar-Quiñonez H, Kaiser L, Martin A, Metz D, Olivares A. Inseguridad alimentaria en latinos de 
California: observaciones de grupos focales. Salud Publica Mex 2003;45:198-205.

16. Wilde PE. Differential response patterns affect foodsecurity prevalence estimates for households with and without Children. J Nutr 2004;134:1910-15.

17. Pérez-Escamilla R, Correa-Segall A, Kurdian L, Archanjo M, Marín-León L, Panigassi G. An adapted version of the U.S. department of agriculture food insecurity module is a valid tool for assessing household food insecurity in Campinas, Brazil. J Nutr 2004;134:1923-28.

18. Melgar-Quiñonez H, Zubieta AC, Valdez E, Whitelaw B, Kaiser L. Validación de un instrumento para vigilar la inseguridad alimentaria en la Sierra de Manantlán, Jalisco. Salud Publica Mex 2005; 47:413-22.

19. Webb P, Coates J, Frongillo EA, Rogers BL, Swindale A, Bilinsky P. Measuring household food insecurity: Why it's so important and yet so difficult to do. J Nutr 2006;136:1404S-08S.

20. Coates J, Wilde PE, Webb P, Rogers BL, House RF. Comparison of a qualitative and a quantitative approach to developing a household food insecurity scale for Bangladesh. J Nutr 2006; 136: 1420S-30S.

21. Melgar-Quinoñez H, Zubieta AC, MkNelly B, Nteziyaremye A, Gerardo MF, Dunford C. Household food insecurity and food expenditure in Bolivia, Burkina Faso, and the Philippines. J Nutr 2006;136:1431S-37S.

22. Coates J, Frongillo EA, Rogers BL, Webb P, Wilde PE, Houser RF. Commonalities in the experience of household food insecurity across cultures: What are measures missing?. J Nutr 2006; 136: 1438S-48S.

23. Swindale A, Bilinsky P. Development of a universally applicable household food insecurity measurement tool: Process, current status, and outstanding issues. J Nutr 2006;136:1449S-52S.

24. Hackett M, Melgar-Quinoñez H, Alvarez-Uribe MC. Internal validity of a household food security scale is consistent among diverse populations participating in a food supplement program in Colombia. BMC publich Health 2008;8:175.

25. Departamento Nacional de Planeación (DPN). Documento CONPES social 113. Política Nacional De Seguridad Alimentaria y Nutricional (PSAN). Bogotá, Colombia, 2008.

26. Álvarez MC, Estrada A, Montoya EC, MelgarQuinoñez H. Validación de la escala de la seguridad alimentaria domestica en Antioquia, Colombia. Salud Publica Mex 2006;48:474-81.
27. Instituto Colombiano de Bienestar Familiar (ICBF). Encuesta Nacional de la Situación Nutricional en Colombia, 2005. Bogotá, Colombia, 2005.

28. BGA Commission on nutritional epidemiology. Recommendations for the design and analysis of nutritional epidemiologic studies with measurement errors in the exposure variables. Eur J Clin Nutrition 1993;47:S53-S57.

29. Chmura H. Evaluating medical test. London. SAGE Publications, 1992.

30. Bland JM, Altman DG. Validating scales and indexes. BMJ 2002;321:606-7.

31. Hair JF, Anderson RE, Tatham RL, Black WC. Análisis multivariante. Quinta edición, Madrid. Prentice Hall, 1999.

32. Kline P. An easy guide to factor analysis. London. Routledge Press, 1994;157-84.

33. Gliem JA, Gliem RR. Calculating, interpreting, and reporting Cronbach's alpha coefficient for likert. type scales. Presented at the Midwest research to practice conference in adult, continuing, and community education. The Ohio State University, Columbus, OH, October 8-10, 2003. Disponible en; www.alumni-osu.org/midwest/midwest\%20papers/ Gliem\%20\&\%20Gliem--Done.pdf. Acceso el 10 de febrero de 2006.

34. Bartfay E, Donner A. The effect of collapsing multinomial data when assessing agreement. Int J Epidemiol 2000;29:1070-75.

35. Feinstein AR, Cicchetti DV. High agreement but low Kappa: I. The problems of two paradoxes. J Clin Epidemiol 1990;43:543-49.

36. Byrt T, Bishop J, Carlin JB. Bias, prevalence and Kappa. J Clin Epidemiol 1993;46:423-29.

37. Egglin TK, Feinstein AR. Context bias. A problem in diagnostic radiology. JAMA 1996;276:1752-55.

38. CDC. EpiInfo, versión 6.04d. Epidemiología en ordenadores. Atlanta, Georgia. Enero, 2001

39. StataCorp, 2008. Stata Statistical Software: Release 10.1 Collegue Station, TX: StataCorp LP.

40. BDMP, 1992. Statistical Software Inc: Release 7.

41. STATA. Estimation of means, totals, ratios, and proportions for survey data. Stata Technical Bulletins 1996;6:213-35.

42. Herrán OF, Prada GE, Quintero DC. Ingesta usual de vitaminas y minerales en Bucaramanga, Colombia. Rev Chil Nutr 2007;34:35-44.

43. Herrán OF, Prada GE, Ardila MF. Ingesta usual de macronutrientes y energía en Bucaramanga, Colombia: Análisis de registros de consumo 1998-2003. Rev Chil Nutr 2007;34):307-19.

44. Bautista L, Herrán OF, Jane A Pryer. Develop- 
ment and simulated validation of a food-frequency questionnaire for the Colombian population. Public Health Nutrition 2005:8:181-88.

45. Herrán OF, Gamboa EM, Prada GE. Diseño y eficacia de pruebas para determinar la deficiencia de hierro. Rev Chil Nutr 2006;33:518-26.

46. Herrán OF, Prada GE, Patiño GA. Canasta básica alimentaria e índice de precios en Santander, Co- lombia, 1999-2000. Salud Publica Mex 2003;45:3542.

47. Alvarado BE, Zunzunegui MV, Delisle H. Validación de escalas de seguridad alimentaria y de apoyo social en una población afro-colombiana: aplicación en el estudio de prevalencia del estado nutricional en niños de 6 a 18 meses. Cad Saúde Pública 2005;21:724-36. 